\title{
Erratum: Ground states of two-dimensional tilted dipolar bosons with density-induced hopping [Phys. Rev. A 103, 043333 (2021)]
}

\author{
Chao Zhang $®$, Jin Zhang, Jin Yang, and Barbara Capogrosso-Sansone
}

(Received 2 December 2021; published 5 January 2022)

DOI: 10.1103/PhysRevA.105.019901

In regard to our original paper, we have realized that there was a typographical error in the Monte Carlo code we have used to get the phase diagrams of Fig. 3. As a result, $U / t$ was not always constant and equal to 20 as stated in the paper. We have redone the simulations at $U / t=20$ and noticed that, while the original results are qualitatively correct, there exist quantitative differences in the position of the transition lines. Here, we report the corrected phase diagrams, obtained at fixed $U / t=20$. Below we briefly describe the main quantitative changes in the phase diagrams.

Figure 3(a): Compared with results in Ref. [1], where density-induced hopping is not taken into account, superfluidity is slightly suppressed. This is due to negative density-induced hopping $\left(T_{x} \sim-0.17\right.$ and $\left.T_{y} \sim-0.17\right)$. The superfluid-checkerboard solid (SF-CB) transition happens around $\gamma \sim 0.053$ (equivalent to $V / t \sim 3.27$ ).

Figure 3(b): As in Fig. 3(a), the density-induced hopping parameters are negative $\left(T_{x} \sim-0.15\right.$ and $\left.T_{y} \sim-0.17\right)$. This results from adjusting the scattering length as to keep $U / t$ fixed.

Figure 3(c): The actual values of density-induced hopping are $0.135<\left|T_{y}\right| / t<0.291$ and $0.149<T_{x} / t<0.210$. The stripe solid phase appears at $\gamma \gtrsim 0.0564$. We notice that, within error bars, the $\gamma$ value at which the solid appears is the same as in the case with no density-induced hopping. The supersolid (SS) at half filling exists for $0.0534<\gamma<0.0564$. The stripe supersolid (SSS) away from half filling exists for $0.0564<\gamma<0.068$. We have observed that for low enough doping, the solid order of the SSS is the same as for the SS. For larger doping, instead, we see that stripes are not uniformly spaced. In the figure, we mark the first-order phase transition with the blue solid region which corresponds to the density range for which one would observe phase coexistence.

Figure 3(d): The corrected values of density-induced hopping are $0.113<\left|T_{y}\right| / t<0.127$ and $0.204<T_{x} / t<0.215$. The stripe solid at half filling exists for $\gamma \gtrsim 0.0363$. We notice that the stripe supersolid is also stabilized upon doping the stripe solid

(a)

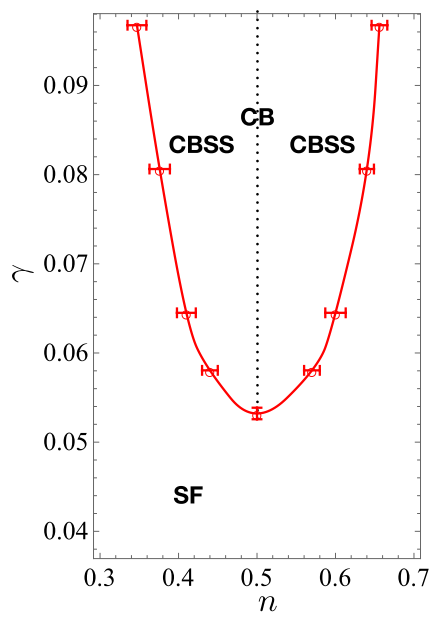

(b) $\quad \theta=11.25^{\circ}$

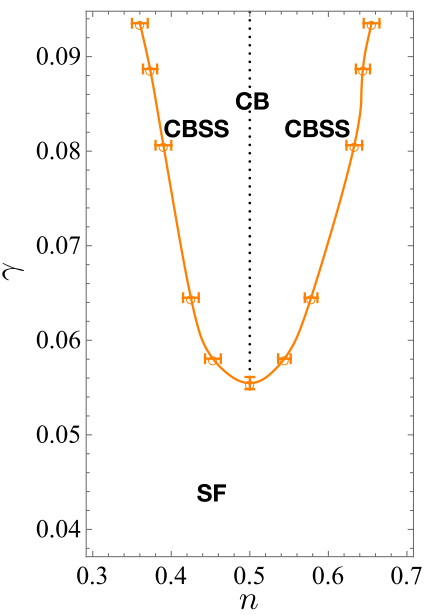

(c)

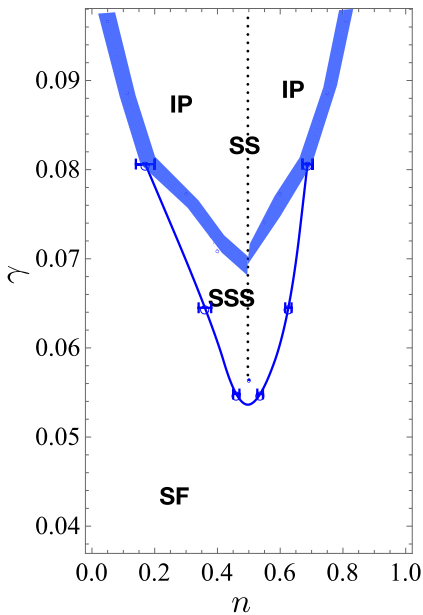

(d) $\quad \theta=67.5^{\circ}$

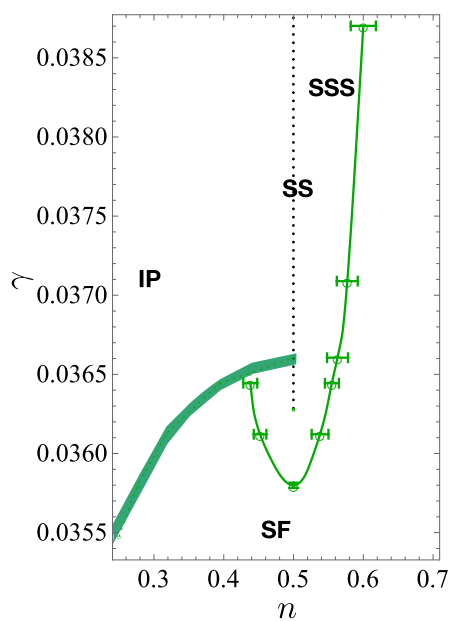

FIG. 3. Amended Fig. 3 in the original paper. Ground-state phase diagram for (a) $\theta=0^{\circ}$, (b) $\theta=11.25^{\circ}$, (c) $\theta=45^{\circ}$, and (d) $\theta=67.5^{\circ}$. The $x$ axis is the filling factor $n$ and the $y$ axis is the dipolar interaction strength $\gamma$. For tilt angles $\theta \lesssim 30^{\circ}$, the solid phase stabilized at half filling corresponds to a checkerboard solid (CB) and the supersolid phase is a CBSS; for $\theta \gtrsim 30^{\circ}$, the solid phase corresponds to a stripe solid (SS) and the supersolid phase is a SSS. IP stands for the incompressible ground states stabilized at rational filling factors. Solid lines correspond to second-order transitions while solid shaded regions correspond to first-order phase transitions. Dotted lines at filling factor $n=0.5$ represent solid phases CB or SS. When not visible, error bars are within symbol size. 


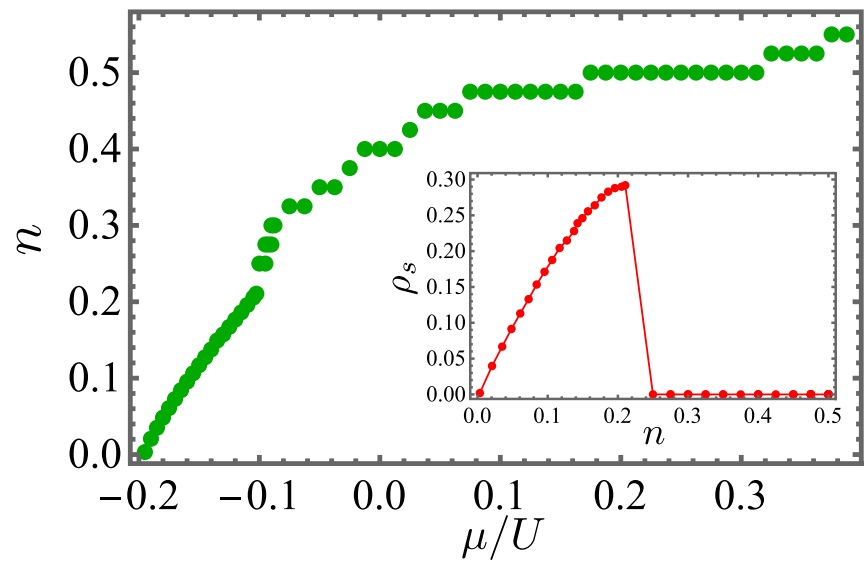

FIG. 4. Amended Fig. 4 in the original paper. Plots correspond to parameters $U / t=20, \theta=45^{\circ}, \gamma=0.081$, and $L=40$. Main plot: Filling factor $n$ as a function of $\mu / U$. Inset: Superfluid density $\rho_{s}$ as a function of filling factor $n$. When not visible, error bars are within symbol size.

with holes, but only at lower $\gamma$ values. The supersolid at half filling exists for $0.0358 \lesssim \gamma \lesssim 0.0363$. Finally, we have marked the first-order phase transition between the incompressible phase and superfluid with a shaded green area.

We have also included amended Figs. 4 and 5.

Finally, in Sec. V, the following statement, "When the lattice constant equals $532 \mathrm{~nm}$, for Cr which has a $\gamma$ of $\sim 0.06$, the superfluid phase should be observed when $\theta=0^{\circ}, 11.25^{\circ}, 45^{\circ}$. When $\theta=67.5^{\circ}$, the incompressible phase, SS phase and SSS phase are realized under different filling conditions," should be replaced with "When the lattice constant equals $532 \mathrm{~nm}$, for $\mathrm{Cr}$ which has a $\gamma$ of $\sim 0.06$, all phases can be stabilized with appropriate choice of filling factor and tilt angle."
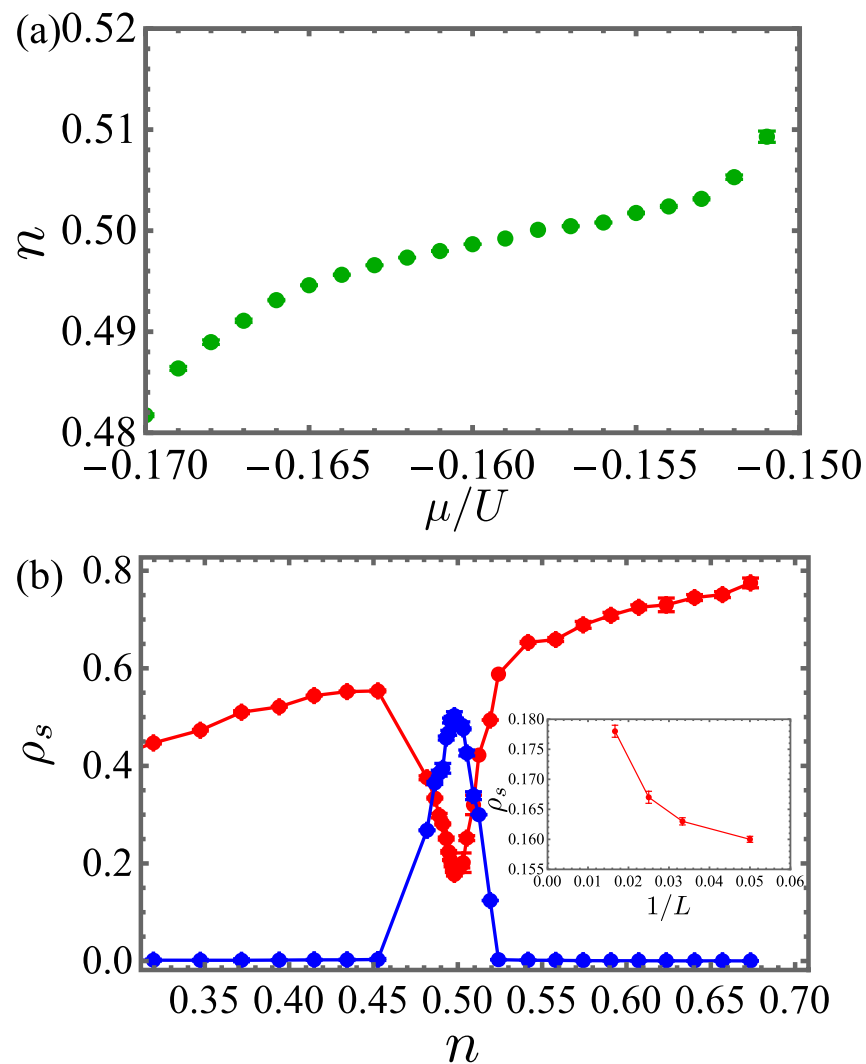

FIG. 5. Amended Fig. 5 in the original paper. Main plots correspond to parameters $U / t=20, \theta=67.5^{\circ}, \gamma=0.0361$, and $L=60$. (a) Filling factor $n$ as a function of $\mu / U$. (b) Superfluid density $\rho_{s}$ and structure factor $S(0, \pi)$ as a function of filling factor $n$. The inset shows the superfluid density $\rho_{s}$ as a function of $1 / L$ at filling factor $n=0.5$. When not visible, error bars are within symbol size. 
The following statement, "For Er, Dy which have larger dipole moments (Er: $\gamma \sim 0.27$, Dy: $\gamma \sim 0.53$ ), different quantum phases other than the superfluid can be realized under different filling conditions at any tilt angle," should be replaced with "For Er, Dy which have larger dipole moments (Er: $\gamma \sim 0.27$, Dy: $\gamma \sim 0.53$ ), different quantum phases can be realized under different filling conditions at any tilt angle."

[1] D. Grimmer, A. Safavi-Naini, B. Capogrosso-Sansone, and S. G. Soyler, Phys. Rev. A 90, 043635 (2014). 